nonverbal communication and imaginative activity, and restricted and repetitive behavior. The PDD and ADHD group had similar scores in communication problems and restricted and repetitive behavior. PDD patients had a higher score than the ADHD group only in the social interaction impairment. In total score, inattention score, and hyperactivity/impulsivity score on the ADHD Rating Scale-IV, both groups were significantly higher than the control group; ADHD and PDD groups showed similar scores in the three ADHD subtypes. ADHD patients had many PDD-related symptoms, and PDD patients had many ADHD-related symptoms. Diagnoses of ADHD and PDD were difficult to distinguish when using the DSMIV criteria, and an individual patient should be evaluated using both questionnaires. (Hattori $\mathrm{J}$, Ogino $\mathrm{T}$, Abiru $\mathrm{K}$, et al. Are pervasive developmental disorders and attentiondeficit/hyperactivity disorder distinct disorders? Brain Dev June 2006;28:371-374). (Respond: Dr Junri Hattori, Department of Child Neurology, Okayama University Graduate School of Medicine and Dentistry, Shikata-cho 2-5-1, Okayama 700-8558< Japan).

COMMENT. Although patients with PDD, according to DSM-IV criteria, were excluded from the ADHD group, the ASSQ total score in the ADHD group was higher than in the control group. Also, all three behavioral category scores in the PDD diagnosis (social interaction, communication problem, and restricted and repetitive behavior) were higher in the ADHD group than in the control group. The ADHD group included subjects whose PDDrelated symptoms did not reach the threshold for PDD diagnosis, based on DSM criteria. Social interaction was the only domain in PDD criteria that was not significantly impaired in the ADHD group, possibly because at least 2 items are required whereas one will suffice in the other domains. In our ADD Neurology Clinic at Children's Memorial Hospital, Chicago, we also have been impressed with the prevalence of symptoms of autism complicating ADHD cases. Sometimes, these symptoms are only apparent after completing a simplified questionnaire for ASD with the parent, leading to more adequate and individualized behavioral and educational intervention. The reported prevalence of autistic spectrum disorder has increased from as few as 4 per 10,000 children in the 1980 s to as many as 50 per 10,000 today (Rivara FP. Arch Pediatr Adolesc Med May 2006;160:548).

\title{
FACTORS THAT INFLUENCE FREQUENCY OF ADHD DIAGNOSIS
}

Factors associated with the probability of attention-deficit/hyperactivity (ADHD) diagnosis among third grade US elementary school children were investigated in a nationally representative sample of 9278 children in the 2002 follow-up of the Early Childhood Longitudinal Survey Cohort conducted at University of Texas, Austin, TX. In a total of $5.44 \%$ children diagnosed with ADHD, the means of several variables differed in frequency of ADHD diagnosis. Variables associated with a lower risk of ADHD were female gender, black children, and Hispanics; children living with a biological father; the western and southern regions vs midwestern US; having a white teacher, relative to a nonwhite teacher; and attending a Catholic vs public school. ADHD diagnosis was more frequent in children born in summer months; in lower-income families; in children taught by an older teacher; and in schools subject to stricter state-level performance accountability laws. Larger class sizes or state laws that restrict school personnel from discussing ADHD treatment options with parents were not influencing factors. (Schneider H, Eisenberg D. Who receives a diagnosis of attention-deficit/hyperactivity disorder in the United States elementary school 
population? Pediatrics April 2006;117:601-609). (Respond: Helen Schneider PhD, Department of Economics, University of Texas at Austin, BRB 3.116, Austin, TX 78712).

COMMENT. The likelihood of ADHD diagnosis is dependent on a child's gender and race, the presence of a biologic father in the home, the teacher's color and age, the season and geographic region of birth, the socio-economic status, and school standards and performance accountability.

\section{EFFECT OF LONG-ACTING STIMULANTS ON GROWTH}

The effect of OROS methylphenidate (OROS MPH, Concerta) [34-43 mg daily; 1.1$1.2 \mathrm{mg} / \mathrm{kg} /$ day] on the height and weight of 178 children, ages 6 to 13 years, treated for at least 21 months for attention-deficit/hyperactivity disorder (ADHD) was evaluated at the Massachusetts General Hospital, Boston. Subjects ( $86 \%$ having received stimulant therapy previously) were at the expected height for their age at baseline and somewhat heavier than expected. Height and weight were measured monthly during the first year of treatment and then every 3 months. Height steadily increased throughout the study, but subjects were on average $0.23 \mathrm{~cm}$ less than expected at month 21 . Weight showed no increase and body mass index (BMI) decreased slightly in the first 4 months of treatment; weight and BMI remained constant after 4 months, and were on average $1.23 \mathrm{~kg}$ less than expected at 21 months. Those taking drug holidays ( $29 \%$ omitting treatment for $>30$ days vs $71 \%$ for $<30$ days during the study) showed trends toward lesser decreases in weight and growth. Among 167 subjects who discontinued treatment early, $36(8.8 \%)$ reported lack of effectiveness, $31(7.6 \%)$ adverse events, $29(7.1 \%)$ lost to follow-up, and $28(6.9 \%)$ failed to follow protocol. Growth measurements were not obtained at both baseline and 21 months in 51. (Spencer TJ, Faraone $\mathrm{SV}$, Biederman J, et al. Does prolonged therapy with a long-acting stimulant suppress growth in children with ADHD? J Am Acad Child Adolesc Psychiatry May 2006;45:527-537). (Respond: Dr Thomas J Spencer, Massachusetts General Hospital, 15 Parkman Street, WACC 725, Boston, MA 02114).

COMMENT. Although the authors conclude that the effects of prolonged OROS MPH on growth of ADHD children are clinically insignificant, and significant decreases in weight are limited to the first months of therapy, their data show some small adverse effects in the group as a whole. The results of this study should not negate the importance of monitoring children carefully for more significant potential effects on growth in the individual child. Furthermore, the possible benefits of drug holidays in lessening long-term adverse effects should not be dismissed by the results of this study; a drug holiday of only $5 \%$ of the time, as used by the authors, is considerably shorter than the 130 day (35\%) annual drug holiday (on weekends and vacations), frequently used in conservative regimens.

Effects of MPH vs mixed amphetamine salts (Adderall) on height in children with ADHD treated for 1 to 3 years were similar; linear regression found a small but statistically significant negative effect on height. Both drugs caused a significant decrease in weight and BMI, patients becoming thinner over time, and this effect was more pronounced with mixed amphetamine salts (Pliszka SR, et al. J Am Acad Child Adolesc Psychiatry May 2006;45:520-526). 